\title{
Neurotropin abrogates lidocaine-induced suppression of neurite growth in cultured rat spinal neurons
}

\author{
Risa Isonaka*, Takashi Katakura and Tadashi Kawakami \\ Department of Physiology, Kitasato University School of Medicine, Kitasato, Minami-ku, Sagamihara, Kanagawa, Japan
}

\begin{abstract}
Background: Neurotropin is an analgesic agent used for the treatment of chronic and neuropathic pain. The effects of this drug have been investigated in clinical practice, and have been recently the focus of attention with respect to its neuroprotective effects on neuropathy caused by anticancer agents. Lidocaine is used to induce local anesthesia and is known to relieve neuropathic pain, but is also known to have neurotoxic effects, causing neurodegeneration after spinal anesthesia. In this study, we used the simple preparation of cultured rat spinal neurons to examine the effects of neurotropin and lidocaine on neurite growth and the effects of neurotropin on lidocaine-induced neurotoxicity.
\end{abstract}

Methods: Spinal neurons were identified by neurofilaments immunostaining and neurite growth of individual neurons was measured using NIH Image software on the captured images.

Results: Neurotropin alone had not apparent effect on neurite growth, whereas lidocaine inhibited neurite growth and induced morphological changes in neurites. Neurotropin abrogated lidocaine-induced suppression of neurite growth in spinal neurons.

Conclusions: This is the first report to show the neuroprotective effects of neurotropin against lidocaine-induced neurotoxicity in spinal neurons. The in vitro results are encouraging to plan further experiments to test this property of neurotropin in laboratory animals.

\begin{abstract}
Abbreviations: TNS: transient neurologic symptoms; NU: neurotropin unit; NGF: nerve growth factor; FITC: fluorescein isothiocyanate; PBS: phosphate buffered saline
\end{abstract}

\section{Introduction}

Neurotropin is an analgesic agent used for the treatment of neuropathic pain, such as lumbago, neck-shoulder-arm syndrome, postherpetic neuralgia [1,2] and fibromyalgia [3], as well as dysesthesia of subacute mylo-optico-neuropathy [4]. The National Institute of Nursing Research has examined the effectiveness of neurotropin in the treatment of chronic pain after limb or large nerve injury since 2000. Recently, Zhang, et al. [5] reported that neurotropin protects against neuropathic pain induced by oxaliplatin, which is the first-line colorectal cancer chemotherapeutic agent, in patients with stage II and III cancers. In this regard, one animal and cell culture study [6] also demonstrated that neurotropin relieves oxaliplatin-induced mechanical allodynia and inhibits oxaliplatin-induced neurite degeneration. Kawashiri, et al. [7] also showed that neurotropin can ameliorate allodynia in rats and axonal degeneration induced by paclitaxel, an anticancer agent used for ovarian cancer, non-small cell lung carcinoma and breast cancer, in cultured pheochromocytoma cell line and rat dorsal root ganglion neurons, without affecting their chemotherapeutic actions. These studies suggest that neurotropin has not only analgesic effects but also neuroprotective effects.

In addition to its anesthetic properties [8,9], lidocaine also has analgesic properties that can relief neuropathic pain, such as peripheral nerve injury [10], spinal cord injury [11], and postherpetic neuralgia [12]. The clinical use of local anesthetics can cause transient neurologic symptoms (TNS) [13,14] following spinal anesthesia. The risk of developing TNS after spinal anesthesia with lidocaine is significantly higher than with other anesthetics such as bupivacaine, prilocaine, and procaine $[15,16]$. Lidocaine is known to have neurotoxic effects and can cause neurodegeneration both in vitro [17-20] and in vivo [21,22].

The present study was designed to examine the effects of neurotropin and lidocaine on neurite growth and to determine whether neurotropin interacts with lidocaine-induced neurotoxicity. We used the simplified preparation of cultured spinal neurons of neonatal rats in order to test the effect of these two agents without any contamination by other inputs known to modulate spinal neuronal activity and drugdrug interaction.

\section{Methods}

\section{Primary cell culture}

The experimental protocol was approved by the Animal Experimentation and Ethics Committee of Kitasato University School of Medicine (\#2013-148 and 2014-077), which conformed to the National Institutes of Health guidelines on the ethical use of animals. On day 20 of pregnancy ( $\sim 1$ day before birth), Wistar rats were anesthetized with pentobarbital sodium $(50 \mathrm{mg} / \mathrm{kg})$ and embryos were removed. The

*Correspondence to: Risa Isonaka, Ph.D, Department of Physiology, Kitasato University School of Medicine 1-15-1 Kitasato, Minami-ku, Sagamihara, Kanagawa, 252-0374, Japan, Tel: +81-42-778-9159; Fax: +81-42-778-9841; Email: isonaka@med.kitasato-u.ac.jp

Key words: neurotropin, lidocaine, neurite growth, spinal neurons

Received: September 04, 2018; Accepted: September 14, 2018; Published: September 17, 2018 
embryonic spinal cord was dissected out and immediately immersed in ice-cold Leibovitz's L-15 medium containing $0.5 \mathrm{mM}$ L-glutamine, and then enzymatically and mechanically dissociated, as described in detail in our previous study [23]. The isolated cells were plated onto poly-L-lysine-coated glass coverslips $(30 \times 40 \mathrm{~mm}, 50 \mu \mathrm{m}$-thickness $)$ and cultured in neurobasal medium (Life Technologies, Grand Island, $\mathrm{NY}$ ) containing $0.5 \mathrm{mM} \mathrm{L}$-glutamine and $2 \% \mathrm{~B}-27$ supplement (Life Technologies) at $37^{\circ} \mathrm{C}$ in a humidified $5 \% \mathrm{CO}_{2}$ atmosphere.

\section{Solution and drugs}

Immediately before each experiment, lidocaine hydrochloride monohydrate (Sigma-Aldrich, St. Louis, MO) and neurotropin ${ }^{\circ}$ (Nihon-Zoki Pharmaceutical Co., Osaka, Japan) were diluted with the culture medium to preselected concentrations. The concentration of neurotropin was expressed in neurotropin unit $(\mathrm{NU}) / \mathrm{ml}$. The $\mathrm{pH}$ of all solutions was adjusted to 7.3-7.4.

\section{Drug treatment}

Spinal cord neuronal cells were incubated in the culture medium for $24 \mathrm{~h}$ and subsequently treated with neurotropin (at final concentrations of $0,0.1,1,10,100$, or $1000 \mathrm{mNU} / \mathrm{ml})$, lidocaine $(0,0.001,0.01,1,10$, or $100 \mathrm{mM}$ ), or the combination of $1 \mathrm{mM}$ lidocaine and neurotropin at each concentration for $72 \mathrm{~h}$.

\section{Immunocytochemistry}

Cells were fixed with $4 \%$ paraformaldehyde for $10 \mathrm{~min}$ and then treated with a protein-blocking agent (Immunon, Pittsburgh, PA) to block non-specific binding sites. The cells were then incubated with mouse anti-phosphorylated neurofilaments antibody (dilution, 1:1000; SMI-31; Convance, Princeton, NJ) for $60 \mathrm{~min}$, followed by fluorescein isothiocyanate (FITC)-conjugated goat anti-mouse IgG secondary antibody (dilution, 1:50; Organon Teknika Corp, Durham, NC) for 60 min. The immunostained cells were washed with PBS and examined using a confocal laser scanning microscope (model LSM710, Zeiss, Germany). Images were linearly adjusted for brightness and contrast with the Zen imaging software (Zeiss). All immunocytochemical procedures were performed at $24^{\circ} \mathrm{C}$.

\section{Measurement and analysis of neurite length}

The length of the individual neurites was measured at $72 \mathrm{~h}$ after treatment with drugs using NIH ImageJ software version 1.44 (http:// rsb.info.nih.gov/ij/index.html) on the captured images. The neurite length in the drug-treated neurons was compared with the control untreated neurons, and the length in a combination of lidocaine and neurotropin-treated neurons was compared with the nonneurotropin-treated neurons by the Steel test for nonparametric multiple comparisons.

\section{Results}

\section{Effects of neurotropin on neurite growth of spinal neurons}

First, we investigated the effects of neurotropin on neurite growth in cultured rat embryo spinal neurons. Figure $1 \mathrm{~A}$ and $1 \mathrm{~B}$ show the effects of neurotropin on the growth of neurites cultured in the absence of neurotropin (control, Figure 1A) and in the presence of $1000 \mathrm{mNU} / \mathrm{ml}$ neurotropin (Figure 1B) for $72 \mathrm{~h}$. Quantitative analysis confirmed that incubation with neurotropin for $72 \mathrm{~h}$ did not change the neurite length, even at the highest concentration (Figure $1 C, P>0.05$ ). Neurotropin did not induce any apparent morphological changes in the cell bodies or neurites, relative to control neurons.

\section{Effects of lidocaine on neurite growth of spinal neurons}

In the next step, we examined the effects of lidocaine on neurite growth. As shown Figure 2A, incubation with lidocaine at the highest
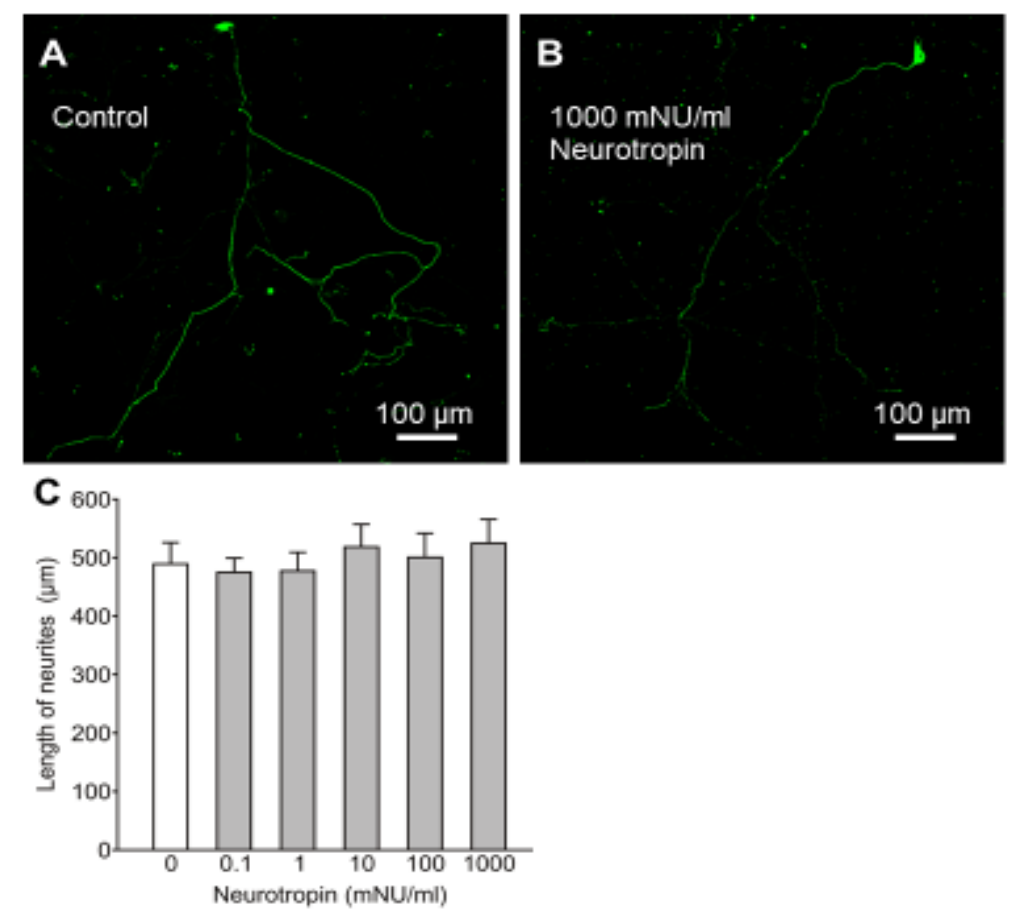

Figure 1. Effects of neurotropin on neurite growth in cultured rat embryo spinal neurons. Photomicrographs of immunostained neurofilaments showing neurite growth of neurons treated for $72 \mathrm{~h}$ with neurotropin at $0 \mathrm{mNU} / \mathrm{ml}$ (control, A) and $1000 \mathrm{mNU} / \mathrm{ml}$ (B). The mean length of neurites after treatment for $72 \mathrm{~h}$ with neurotropin $(\mathrm{C})$. Data are mean $\pm \mathrm{SEM}$ of $>50$ independent samples in each group. $P>0.05$ (vs. control), Steel test 
concentration $(100 \mathrm{mM})$ resulted in damage of neurites, compared with the control (Figure 1A). Quantitative analysis confirmed that neurite length under $1,10,100 \mathrm{mM}$ lidocaine for $72 \mathrm{~h}$ was significantly less than the control (Figure $2 \mathrm{~B}, P<0.005$ ). These results indicate that lidocaine inhibits neurite growth of spinal neurons, and at the highest concentration it caused morphological changes in neurites.

\section{Effects of neurotropin on lidocaine-induced suppression of neurite growth}

We investigated the effects of neurotropin on lidocaine-induced suppression of neurite growth. As shown in Figure 3A and 3B, treatment with $1000 \mathrm{mNU} / \mathrm{ml}$ neurotropin and $1 \mathrm{mM}$ lidocaine abrogated the growth inhibitory effect of lidocaine. Neurons cultured for $72 \mathrm{~h}$ with a combination of neurotropin $(>1 \mathrm{mNU} / \mathrm{ml})$ and lidocaine $(1 \mathrm{mM})$ had significantly longer neurites compared to the mean length of spinal neurons treated with lidocaine alone (Figure 3C, $P<0.05$ ). These results highlight the neuroprotective effect of neurotropin against the growth inhibitory effect of lidocaine.

\section{Discussion}

The present study showed that neurotropin had no apparent effect on neurite growth and also did not cause morphological changes in cultured rat embryo spinal neurons even when used at the highest concentration $(1000 \mathrm{mNU} / \mathrm{ml})$. In contrast, lidocaine inhibited neurite growth of spinal neurons and induced morphological damage at higher concentrations. Furthermore, neurotropin abrogated neurite growth suppression and morphological changes induced by lidocaine.

Recently, Fukuda, et al. [24] reported that neurotropin enhances neuritogenesis by promoting tyrosine kinases receptor-mediated nerve growth factor (NGF) in a pheochromocytoma cell line. They also showed the lack of definite neurotropin-induced enhancement of neurite growth in the absence of NGF, suggesting that the action of neurotropin requires the presence suboptimal concentrations of NGF. On the other hand, Taneda, et al. [25] suggested that neurotropin inhibits NGF-induced neurite outgrowth in cultured rat dorsal root ganglion neurons. Both anti-NGF antibodies and NGF receptor inhibitors
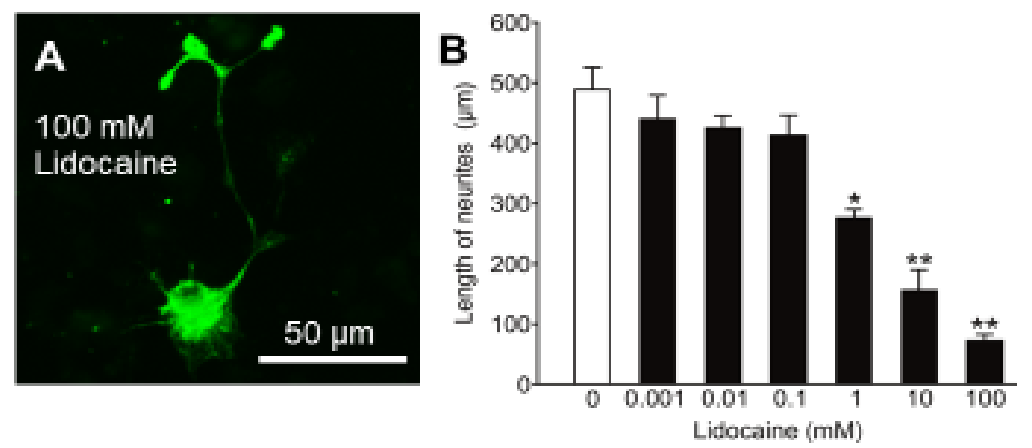

Figure 2. Effects of lidocaine on neurite growth in cultured rat embryo spinal neurons. Photomicrograph of immunostained neurofilaments showing neurite growth of neurons cultured in the presence of $100 \mathrm{mM}$ lidocaine for $72 \mathrm{~h}$ (A). The mean length of neurites after treatment for $72 \mathrm{~h}$ with lidocaine (B). Data are mean \pm SEM of $>50$ independent samples in each group. ${ }^{*} \mathrm{p}<0.005,{ }^{*} \mathrm{p}<0.0005$ (vs control group), Steel test
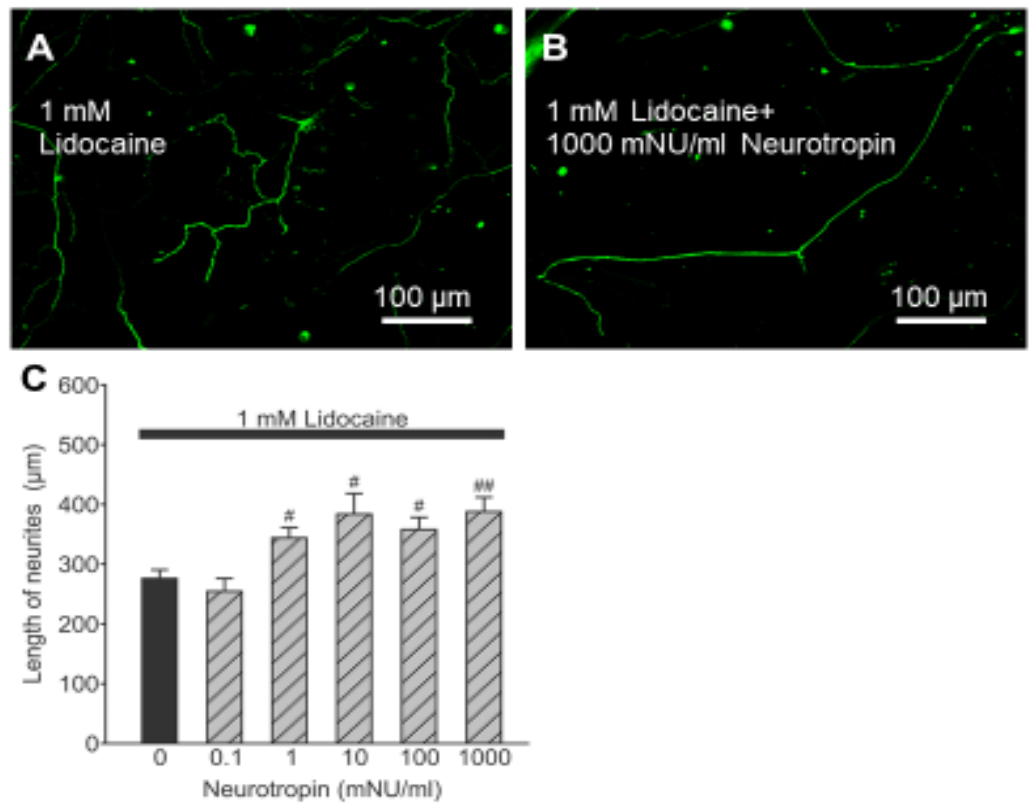

Figure 3. Effects of neurotropin on lidocaine-induced suppression of neurite growth of spinal neurons. Photomicrographs of immunostained neurofilaments showing neurite growth of spinal neurons treated with $1 \mathrm{mM}$ lidocaine alone (A) and a combination of $1 \mathrm{mM}$ lidocaine and $1000 \mathrm{mNU} / \mathrm{ml}$ neurotropin for $72 \mathrm{~h}$ (B). The mean length of neurites after treatment for 72 $\mathrm{h}$ with a combination of lidocaine at $1 \mathrm{mM}$ and neurotropin for $72 \mathrm{~h}(\mathrm{C})$. Data are mean $\pm \mathrm{SEM}$ of $>50$ independent samples in each group. ${ }^{\#} \mathrm{p}<0.05$, ${ }^{\#} \mathrm{p}<0.005$, (vs. non-neurotropin-treated neurons), Steel test 
significantly inhibited the sprouting of epidermal nerve fibers as well as the scratching behavior of atopic ND/Nga mice. Based on these findings, they suggested that neurotropin has antipruritic effects due to inhibition of NGF-induced neurite outgrowth. Intriguingly, although the above studies reported different results, both demonstrated that the action of neurotropin on neurite growth requires NGF. However, using growing cultured rat dorsal root ganglion neurons, Kawashiri, et al. $[6,7]$ demonstrated that neurotropin exhibited a neuroprotective effect against anti-cancer agents in the absence of NGF. Based on the latter finding, neurons were cultured in the present study without NGF; in other words, our results described the pure effects of neurotropin on neurite growth with or without lidocaine in rat spinal neurons.

Neurite growth is a basic neuronal process, and it is easily affected by a variety of extracellular events in cultured neurons, therefore, monitoring neurite growth has been useful in investigating neuronal toxicity [26]. First, our findings suggest that neurotropin had no apparent neurotoxicity on spinal neurons. The estimated maximum plasma concentration in patients treated with neurotropin was $4 \mathrm{mNU}$ / $\mathrm{ml}$ [27]. Taken together, the results suggest that neurotropin has little neurotoxicity even at the extremely high concentrations used clinically. This could explain the fewer side effects of neurotropin encountered in clinical practice.

Our results also showed that lidocaine at suboptimal concentrations is neurotoxic; inhibiting neurite growth of spinal neurons. Anderson and Bamburg [28] reported that neurites of the dorsal root ganglion grown in vitro with lidocaine lost their attachments with the substratum and underwent immediate retraction into the cell body. Radwan, et al. [29] demonstrated that local anesthetics, such as lidocaine, induced collapse of filopodia and lamellipodia of the isolated dorsal root ganglion neurons of chick embryos, suggesting that local anesthetics induced morphological changes in growing neurons. These reports indicate degeneration of neurite growth during lidocaine treatment, lending support to our findings.

Finally, our results suggest that neurotropin can protect against lidocaine-induced neurotoxicity. Cui, et al. [30] reported that extracts from rabbit skin inflamed by the vaccinia virus, which contains the same ingredient as neurotropin, attenuate bupivacaine-induced spinal neurotoxicity in pregnant rats. Their histological analysis showed significantly reduced number of apoptotic cells in the extractspretreatment group compared with bupivacaine-treated group in rat dorsal root ganglion. Considered together with our findings, we speculate that neurotropin can potentially provide protection for spinal neurons against neuronal damage induced by not only lidocaine but also another local anesthetics. However, using our simple in vitro preparation, we cannot state affirmatively that neurotropin protects against the side effects of anesthesia, and therefore further animal experiments followed by clinical studies are needed to confirm these findings.

In conclusion, the present study using cultured rat spinal neurons demonstrated that neurotropin is far less neurotoxic to neurite growth in spinal neurons than lidocaine. Furthermore, neurotropin protected against lidocaine-induced inhibition of neurite growth. These findings suggest that neurotropin could be potentially useful clinically in preventing lidocaine neurotoxicity. The results could also help in the design of new and more effective and safe therapies that enhance the safety of local anesthetics.

\section{Disclosures}

R.I. conceived and designed the experiments, also performed the experiments, analyzed the data, and wrote the paper. T.K. assisted with animal experiments and analyzing data. T.K. assisted with the designing the study and interpreting results.

\section{Acknowledgments}

This work was supported by JSPS Grant-in-Aid for Young Scientist (B) (Grant \#24791624) and a grant from Kitasato University School of Medicine, Japan.

\section{Competing interests}

The authors declare no conflict of interest.

\section{References}

1. Nishiyama T, Matsukawa T, Yamashita K (2006) Comparison between neurotropin and mepivacaine for stellate ganglion injection. J Anesth 20: 240-242. [Crossref]

2. Yamamura H, Dan K, Wakasugi B, Kiyono S, Hyodo M, et al. (1998) Effect of neurotropin on postherpetic neuralgia. Igakuno-Ayumi 147: 651-664.

3. Toda K, Tobimatsu Y (2008) Efficacy of neurotropin in fibromyalgia: a case report Pain Med 9: 460-463. [Crossref]

4. Sobue I, Tashiro K, Hanakago R, Ando K, Yamada T, et al. (1992) Clinical evaluation of neurotropin injections on dysesthesia of SMON (subacute myelo-optico-neuropathy) - A multi-institutional double-blind comparative study. J Clin Therap Med 8: 833-851.

5. Zhang RX, Lu ZH, Wan DS, Wu XJ, Ding PR, et al. (2012) Neuroprotective effect of neurotropin on chronic oxaliplatin-induced neurotoxicity in stage II and stage III colorectal cancer patients: results from a prospective, randomised, single-centre, pilot clinical trial. Int J Colorectal Dis 27: 1645-1650.

6. Kawashiri T, Egashira N, Watanabe H, Ikegami Y, Hirakawa S, et al. (2011) Prevention of oxaliplatin-induced mechanical allodynia and neurodegeneration by neurotropin in the rat model. Eur J Pain 15: 344-350.

7. Kawashiri T, Egashira N, Itoh Y, Shimazoe T, Ikegami Y, et al. (2009) Neurotropin reverses paclitaxel-induced neuropathy without affecting anti-tumour efficacy. Eur $J$ Cancer 45: 154-163.

8. Gordh T (1949) Xylocain, a new local analgesic. Anaesthesia 4: 4-9. [Crossref]

9. Phillips OC, Ebner H, Nelson AT, Black MH (1969) Neurologic complications following spinal anesthesia with lidocaine: a prospective review of 10,440 cases. Anesthesiology 30: 284-289.

10. Attal N, Rouaud J, Brasseur L, Chauvin M, Bouhassira D (2004) Systemic lidocaine in pain due to peripheral nerve injury and predictors of response. Neurology 62: 218-225. [Crossref]

11. Hagen EM, Rekand T (2015) Management of Neuropathic Pain Associated with Spinal Cord Injury. Pain Ther 4: 51-65. [Crossref]

12. Binder A, Bruxelle J, Rogers P, Hans G, Bösl I, et al. (2009) Topical 5\% lidocaine (lignocaine) medicated plaster treatment for post-herpetic neuralgia: results of a double-blind, placebo-controlled, multinational efficacy and safety trial. Clin Drug Investig 29: 393-408. [Crossref]

13. Drasner K (2002) Local anesthetic neurotoxicity: clinical injury and strategies that may minimize risk. Reg Anesth Pain Med 27: 576-580.

14. Pollock JE (2003) Neurotoxicity of intrathecal local anaesthetics and transient neurological symptoms. Best Pract Res Clin Anaesthesiol 17: 471-484.

15. Hampl KF, Schneider MC, Ummenhofer W, Drewe J (1995) Transient neurologic symptoms after spinal anesthesia. Anesth Analg 81: 1148-1153.

16. Zaric D, Pace NL (2009) Transient neurologic symptoms (TNS) following spinal anaesthesia with lidocaine versus other local anaesthetics. Cochrane Database Syst Rev: CD003006. [crossref]

17. Bainton CR, Strichartz GR (1994) Concentration dependence of lidocaine-induced irreversible conduction loss in frog nerve. Anesthesiology 81: 657-667.

18. Gold MS, Reichling DB, Hampl KF, Drasner K, Levine JD (1998) Lidocaine toxicity in primary afferent neurons from the rat. J Pharmacol Exp Ther 285: 413-421. [Crossref] 
19. Kanai Y, Katsuki H, Takasaki M (1998) Graded, irreversible changes in crayfish giant axon as manifestations of lidocaine neurotoxicity in vitro. Anesth Analg 86: 569-573.

20. Kanai Y, Katsuki H, Takasaki M (2000) Lidocaine disrupts axonal membrane of rat sciatic nerve in vitro. Anesth Analg 91: 944-948. [Crossref]

21. Kishimoto T, Bollen AW, Drasner K (2002) Comparative spinal neurotoxicity of prilocaine and lidocaine. Anesthesiology 97: 1250-1253.

22. Sakura S, Kirihara Y, Muguruma T, Kishimoto T, Saito Y (2005) The comparative neurotoxicity of intrathecal lidocaine and bupivacaine in rats. Anesth Analg 101: 541547, table of contents. [Crossref]

23. Isonaka R, Katakura T, Kawakami T (2012) Effect of inhibition of superoxide dismutase on motor neurons during growth: comparison of phosphorylated and nonphosphorylated neurofilament-containing spinal neurons by histogram distribution. Brain Res 1470: 11-16.

24. Fukuda Y, Fukui T, Hikichi C, Ishikawa T, Murate K, et al. (2015) Neurotropin promotes NGF signaling through interaction of GM1 ganglioside with Trk neurotrophin receptor in PC12 cells. Brain Res 1596: 13-21.
25. Taneda K, Tominaga M, Tengara S, Ogawa H, Takamori K (2010) Neurotropin inhibits both capsaicin-induced substance $\mathrm{P}$ release and nerve growth factor-induced neurite outgrowth in cultured rat dorsal root ganglion neurons. Clin Exp Dermatol 35: 73-77.

26. Abdulla EM, Campbell IC (1993) Use of neurite outgrowth as an in vitro method of assessing neurotoxicity. Ann N Y Acad Sci 679: 276-279. [Crossref]

27. Hosoe T, Uekusa H, Shigematsu A, Naka F, Nakamura K, Awazu S (2007) Prediction of drug interactions between neurotropin and clinically used drugs by in vitro methods using radiolabelled substrates. Pharm Regul Sci 38: 369-380.

28. Anderson PL, Bamburg JR (1981) Effects of local anesthetics on nerve growth in culture. Dev Neurosci 4: 273-290. [Crossref]

29. Radwan IA, Saito S, Goto F (2002) The neurotoxicity of local anesthetics on growing neurons: a comparative study of lidocaine, bupivacaine, mepivacaine, and ropivacaine. Anesth Analg 94: 319-324.

30. Cui R, Xu S, Wang L, Lei H, Cai Q, et al. (2013) Extracts from rabbit skin inflamed by the vaccinia virus attenuate bupivacaine-induced spinal neurotoxicity in pregnant rats. Neural Regen Res 8: 983-990.

Copyright: (2018 Isonaka R. This is an open-access article distributed under the terms of the Creative Commons Attribution License, which permits unrestricted use, distribution, and reproduction in any medium, provided the original author and source are credited. 